\title{
Innovative Management Strategy for Combined Proximal Median and UInar Nerve Extensive Injury: A Case Report
}

\author{
Samir Mohamed Ghoraba, IMRCS, MSc*; Sameh Elghamry Elshawadfy, MD; \\ Tarek Gamal Shoukr, MD; Wael Hussein Mahmoud, MD; Hashem Mohamed Ayad, MD \\ Department of Plastic and Reconstructive Surgery, Tanta University, Gharbia, Egypt
}

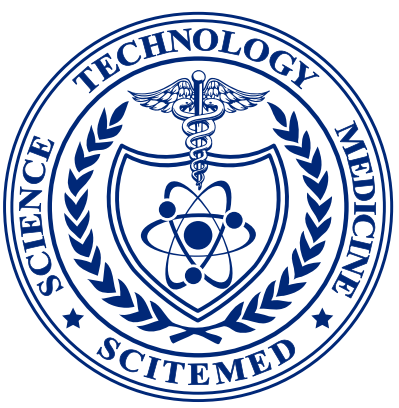

\section{Abstract}

Combined proximal median and ulnar nerve injury is extremely difficult to treat, because a timely successful reinnervation in such proximal injury is unlikely to occur. Here, we used a safe strategy to manage such incapacitating injury in the form of direct transfer of abductor pollicis longus (APL) nerve to the motor fascicles of the ulnar nerve, using the proximal ulnar nerve itself as a graft for the median nerve defect, combined with simultaneous extensor indicis proprius (EIP) opponoplasty. End-to-side transfer of the distal sensory fascicles of the ulnar nerve to the superficial radial nerve at the mid-forearm level was performed. We applied this management strategy to a 14-year-old female who suffered an extensive injury of both median and ulnar nerves at the distal arm level. After 14 months of regular follow up, the patient effectively restored both intrinsic and extrinsic hand functions and variable sensory recovery with minimal morbidity.
\end{abstract}

\section{Introduction}

The highly adaptive function of the hand contributes to the gravity of any kind of nerve injury it sustains. Proximal ulnar nerve injuries seriously impact patient's daily activity. Numerous studies have reported the poor outcomes of the conventional methods of managing such incapacitating injuries [1, 2].

Nerve transfer is a formidable and relatively recent procedure that has been added to the evolving reconstructive armamentarium used to restore the motor function in brachial plexus injuries. Using expendable near donor nerves, nerve transfer techniques conveniently allow the regenerating nerves to travel much shorter distance, and hence it offers a timely successful reinnervation. In terms of restoration of muscle function, the time window between the initial nerve injury and the reinnervation is paramount. One year of denervation initiates an irreversible fibrofatty degeneration of the paralyzed muscles. Through the provision of shorter time of reinnervation, nerve transfer revolutionized the philosophy of nerve surgery [3].

Distal transfer of the terminal anterior interosseous nerve (AIN) to the motor fascicles of the ulnar nerve is a promising new technique in the isolated high ulnar nerve injuries. Nevertheless, the associated high median nerve injury, losing the AIN as a donor nerve, precludes the use of such transfer technique, thus rendering combined proximal median and ulnar nerve injury a challenging encounter that proves its notorious difficulty to treat [4].

We present a case of combined proximal median and ulnar nerves extensive injury that was successfully managed using a novel safe strategy implemented, as follows: (1) direct transfer of abductor pollicis longus (APL) branch of the Posterior interosseous nerve to the motor fascicles of ulnar nerve for restoration of the function of the intrinsic hand musculature; (2) extensor indicis proprius (EIP) opponoplasty; (3) use of the proximal ulnar nerve itself as a non-vascularized nerve graft for the median nerve defect; and (4) end-to-side transfer of the distal sensory fascicles of the ulnar nerve to the superficial radial nerve at the mid-forearm level.

\section{Case Report}

A 14-year-old female suffered a severely lacerated wound on the distal half of the anterior aspect of her left arm. The brachial artery, median and ulnar nerves were extensively lacerated and the overlying skin was devitalized. Examination of the radial nerve revealed normal function.

Debridement of the devitalized soft tissue left a skin defect $(12 \times 9 \mathrm{~cm})$, a median nerve defect $(11 \mathrm{~cm})$, and an ulnar nerve defect $(14 \mathrm{~cm})$. In addition, there was $(8 \mathrm{~cm})$ segmental contusion of the brachial artery (Figure 1).

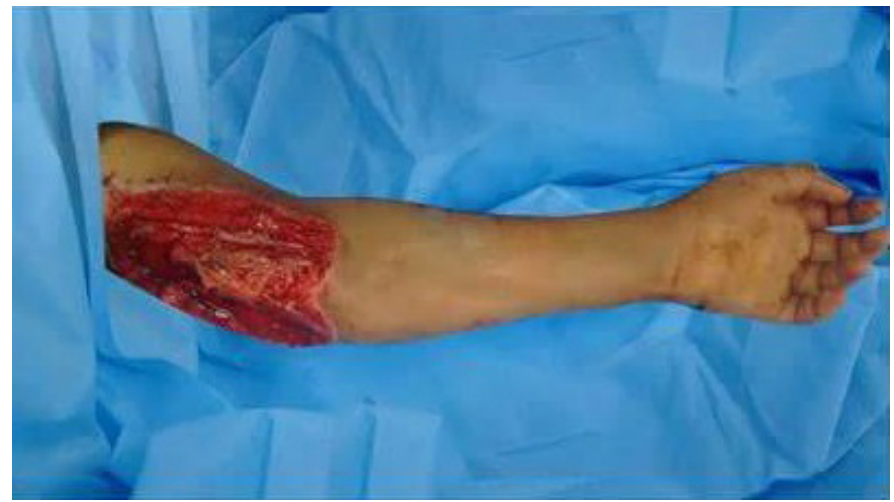

A

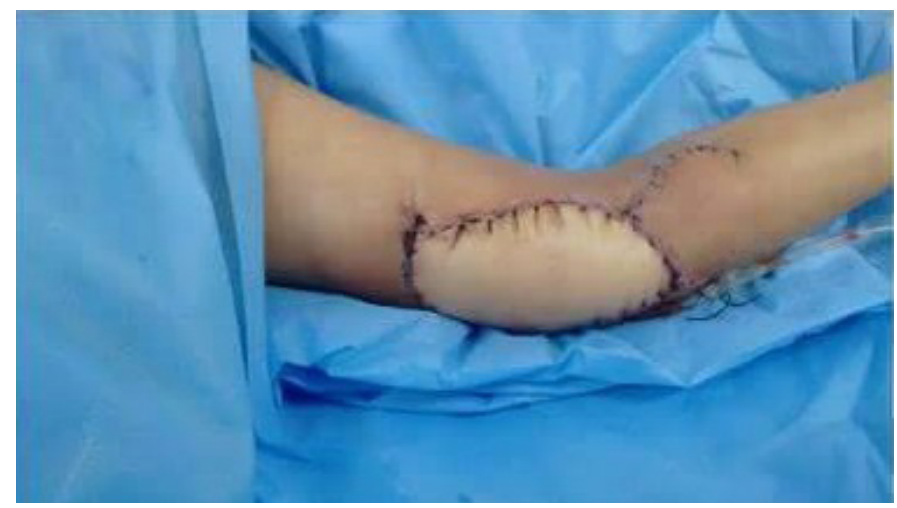

B

Figure 1. (A) The complex soft tissue defect after debridement; (B) Coverage with latissmus dorsi pedicled myocutaneous flap. 
A 2-staged reconstruction strategy was planned and conducted. In the first stage, the brachial artery was reconstructed using an autologous vein graft with a pedicled latissimus dorsi (LD) myocutanous flap to manage the skin defect. In the second stage conducted 2 months later, the nerve injuries were addressed after the soft tissue coverage durability and limb vascularity were ensured. APL nerve was transferred to the motor fascicles of the ulnar nerve at the mid-forearm level in the posterior compartment; meanwhile, the proximal remaining part of the ulnar nerve itself was harvested as an interposition graft for median nerve reconstruction. The distal sensory fascicles of the ulnar nerve were transferred to the superficial radial nerve in the end-to-side pattern at the mid-forearm level. EIP opponoplasty was conducted simultaneously. Eight weeks postoperatively, the patient recovered good thumb opposition and thumb abduction, and the extension were normal, despite of paralysis of the APL. Nine months onwards, the patient gradually regained the motor functions of both median and ulnar nerves. Ranging from protective sensation to good 2-points discrimination, the hand sensation recovered on the final assessment.

\section{Surgical Technique}

Preoperatively, the interval between brachioradialis (BR) and extensor carpi radialis longus (ECRL) muscles was marked at the proximal forearm level and the dorsal skin incision was outlined $5 \mathrm{~cm}$ distal to the lateral epicondyle as an extension of the interval between the 2 muscles (Figure $2 \mathrm{~A})$. Another volar incision was marked along the medial aspect of the middle part of the forearm (Figure 2B).

Under general anesthesia and tourniquet use, the dorsal forearm skin incision was carried out to expose the branch of APL between the BR and ECRL muscle bellies. The posterior interosseous nerve was identified distal to the supinator muscle and traced distally to locate the branch to the APL muscle where it was looped at this point (Figure 3A).

The volar forearm skin incision was done exposing the ulnar nerve trunk at the mid-forearm level, and intraneural microdissection was conducted for identification and isolation of the motor fascicles, which reside between the more medial sensory fibres of the dorsal sensory branch and the more lateral fibers of digital sensation at this specific level (Figure 3B). After isolation of the motor fascicles, a more proximal dissection was done to ensure adequate redundancy for a tension-free neurorraphy in the posterior compartment of the forearm.

At this point, the tourniquet was released for 20 minutes, hemostasis was accomplished, and the nerve stimulator was used to confirm
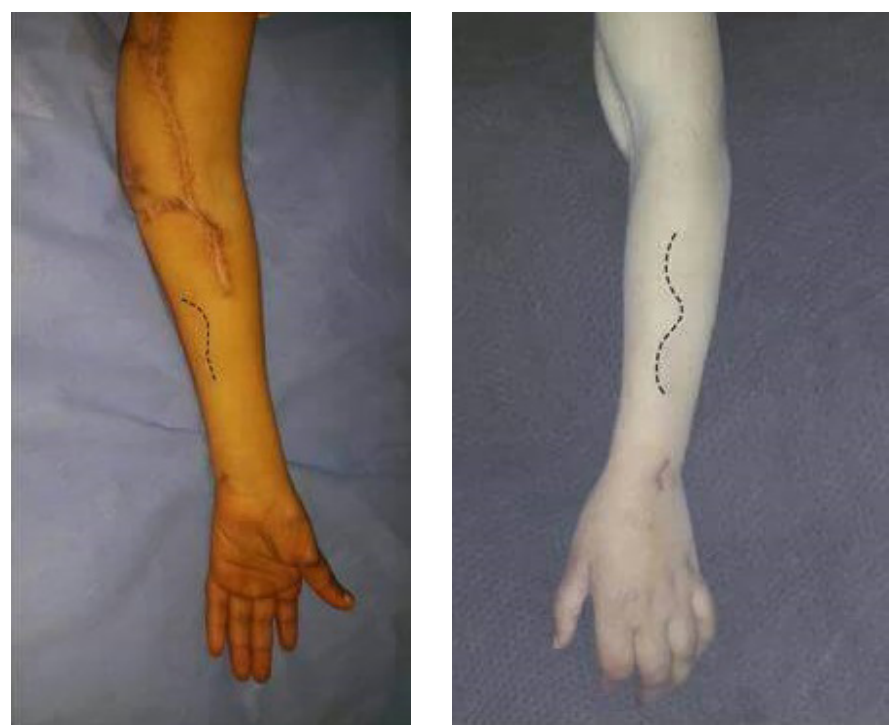

A

B

Figure 2. Preoperative markings. (A) The interval between brachioradialis and extensor carpi radialis longus muscles; (B) Volar incision along the medial aspect of the mid-forearm.

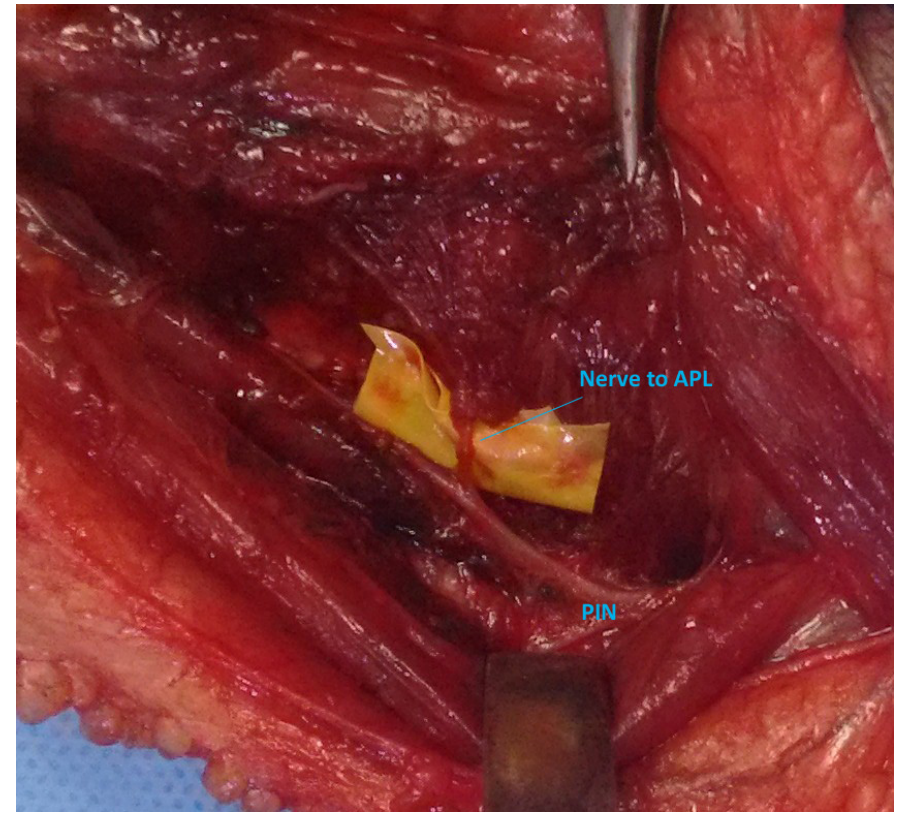

A

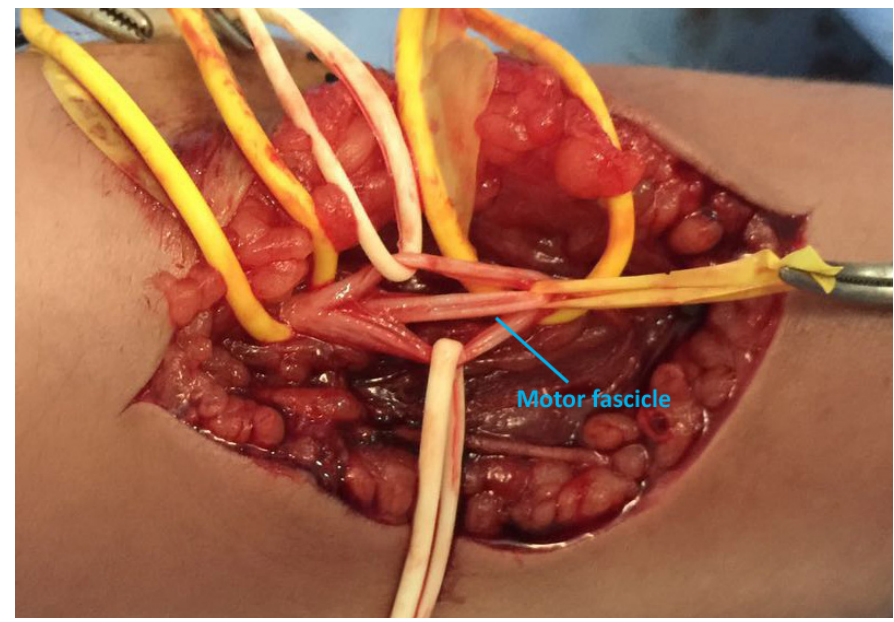

B

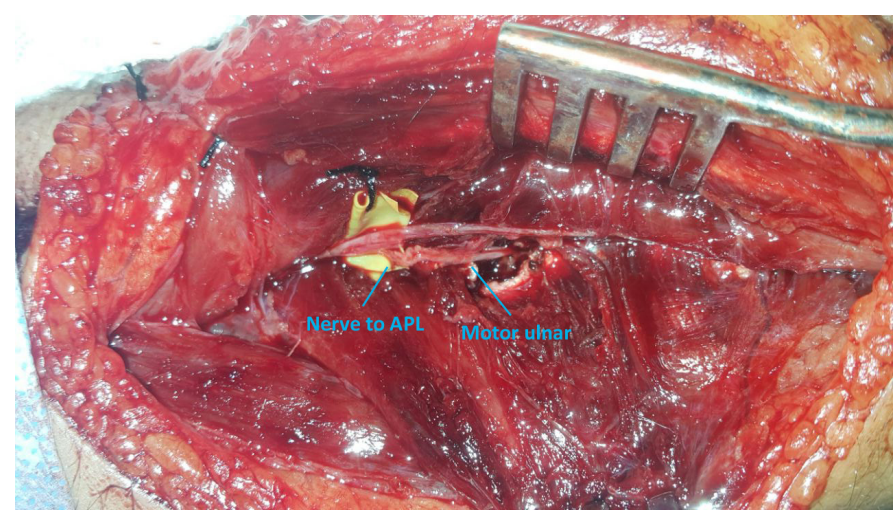

C

Figure 3. (A) Identification of the nerve to abductor pollicis longus muscle; (B) Identification of the motor fascicles of the ulnar nerve at the mid-forearm level; (C) APL to motor ulnar direct nerve transfer. APL, abductor pollicis longus. 
identification of the branch to APL. Using a blunt dissection, a capacious window was created, through which the motor ulnar fascicle was rerouted to reach the posterior compartment. The APL-to-motor ulnar nerve direct transfer was done using 10/0 nylon sutures and augmented with fibrin glue, and tension-free repair was confirmed (Figure 3C). End-to-side transfer of the distal sensory fascicles of the ulnar nerve to superficial radial nerve was conducted through a capacious epineural window using 10/0 nylon sutures.

After tourniquet reapplication, the proximal and distal ends of the median nerve were exposed through two separate incisions (Figure 4A) and the proximal part of the ulnar nerve itself was harvested and tunneled underneath the LD flap to graft the median nerve defect (Figure $4 \mathrm{~B})$. After the closure of the incisions, EIP opponoplasty was conducted (Figure 4C and 4D).

\section{Postoperative Care and Outcomes Assessment}

The upper extremity was immobilized for 3 weeks; elbow in 30 degrees flexion, wrist in modest flexion, metacarpophalangeal joint (MPJ) in 60 degrees flexion, and the thumb in maximum opposition. After three weeks, elbow mobilization was allowed while thumb immobility was continued for further 3 weeks. The final outcomes assessment, 14 months postoperatively, revealed the following: (1) thumb functions were good; the thumb touched the tip of the little finger (Kapandji score 7), in addition to normal extension and abduction, flexor pollicis longus (FPL) motor power was M3 (Figure 5); (2) upon evaluating the hand intrinsic musculature, no clawing was detected and the patient could maintain the intrinsic plus position (Figure 6A); (3) wrist flexors recovered with M3+ muscle power (Figure 6B); (4) finger flexors recovered with M3 muscle power; (5) in spite of weak hand grip, the patient could hold quite small objects (Figure $6 \mathrm{C}$ ); (6) protective sensation was noted in the medial third of palm, ring and little finger while the sensory recovery was better in the lateral three fingers with 2 point discrimination ranging from $7-9 \mathrm{~mm}$ at the finger tips.

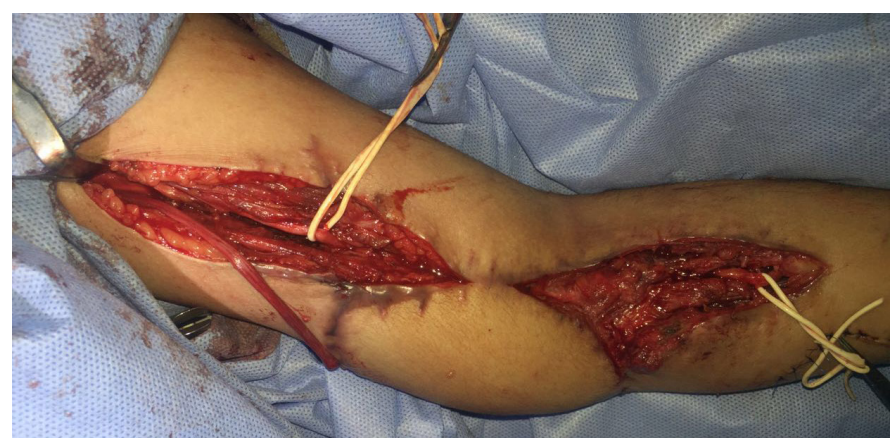

A

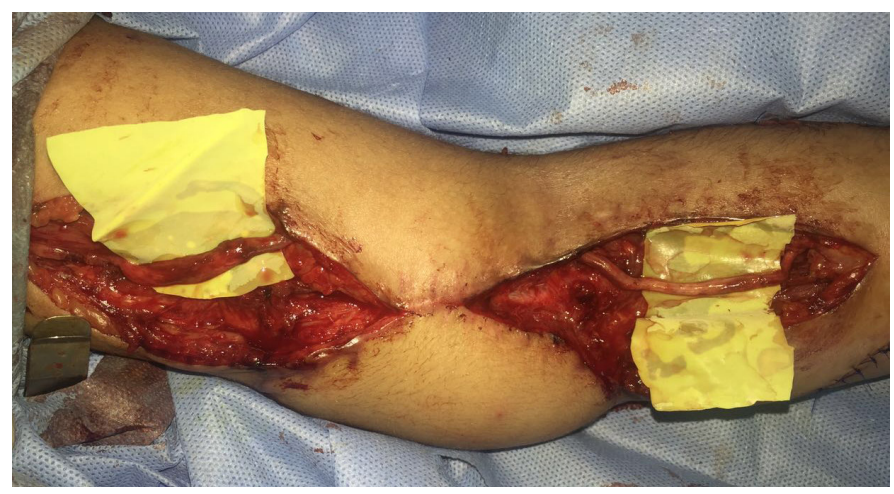

B

Figure 4. (A) Exposure of the proximal and distal ends of the median nerve; (B) Using the proximal ulnar nerve to graft the median nerve defect; (C) Harvest of EIP tendon; (D) Implementation of the EIP opponplasty. EIP, extensor indicis proprius.

\section{Discussion}

A high ulnar nerve injury associated with proximal median nerve injury represents a reconstructive challenge and has a profound detrimental impact on hand function. Conventional nerve reconstruction techniques, either primary neurorraphy or nerve grafts, usually address the deficit of the extrinsic muscles, however, in such proximal ulnar nerve injuries the recovery of the intrinsic muscles of the hand is rare.

Phillips et al. [5] reported a single case of distal expendable radial nerve branches (extensor indicis proprius, abductor pollicis longus, and extensor pollicis brevis) to the deep branch of ulnar nerve direct transfer with good results. However, the clawing was persistent after a year, making the recovery of the ulnar nerve function questionable. Moreover, sacrificing the branch to EIP precludes reliable opponoplasty, which is crucial in such proximal median nerve injury. We believe that the restoration of thumb function in such kind of injuries is a priority.

In this report, we preserved the branch to EIP and performed EIP opponoplasty in the same setting for a better functional outcome. We transferred the APL nerve only to the motor ulnar nerve, and the size mismatch between the donor and recipient nerve was accepted. Using the proximal part of the ulnar nerve itself as a graft for the median nerve, spared the sural nerve and limited the surgery to one region. Offering a new successful solution for the ulnar sensory deficit is substantial for a better outcome.

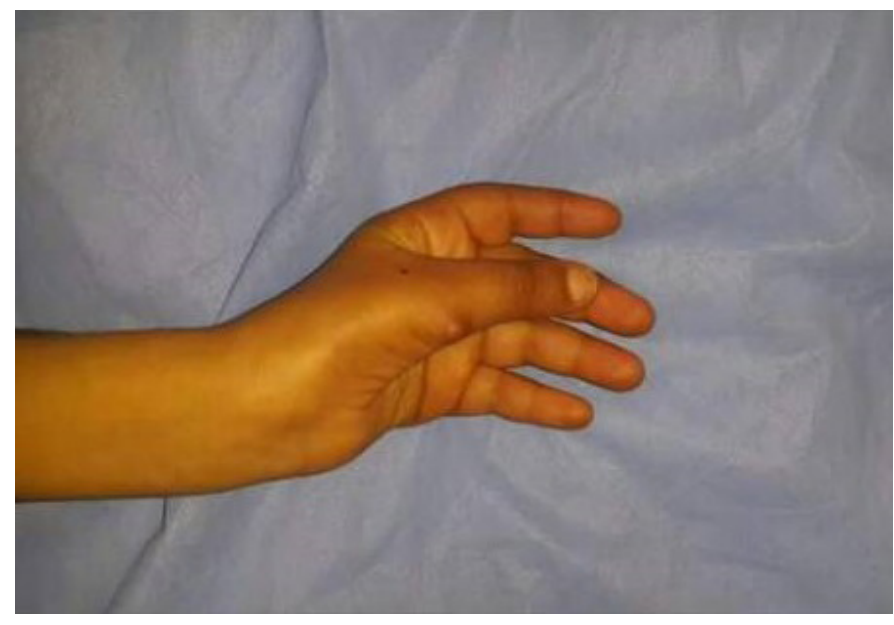

A

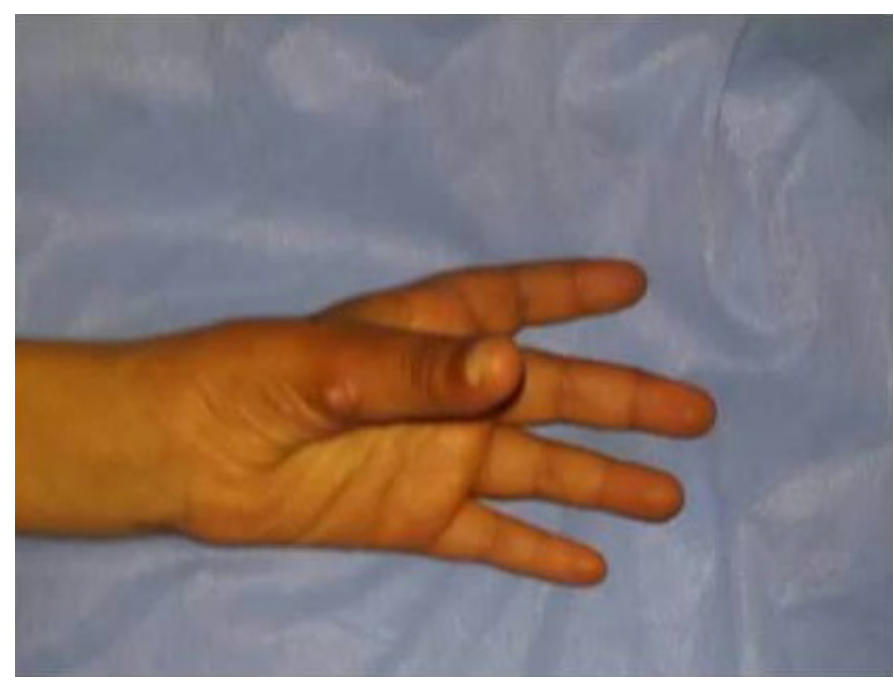

B

Figure 5. (A) Good thumb opposition; (B) Thumb abduction and extension. 


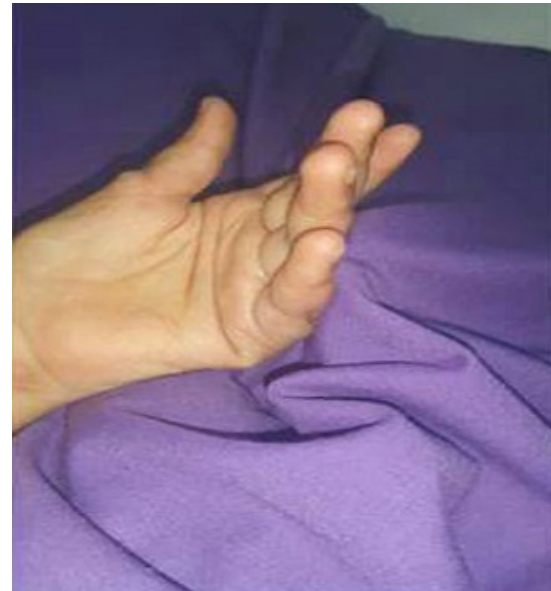

A

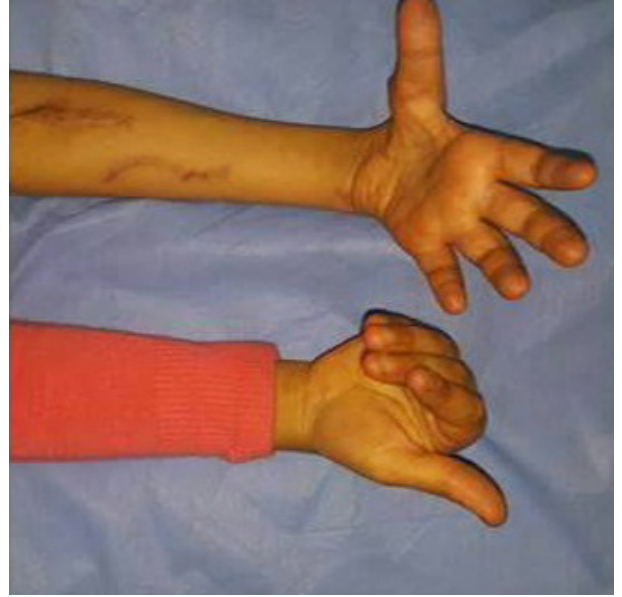

B

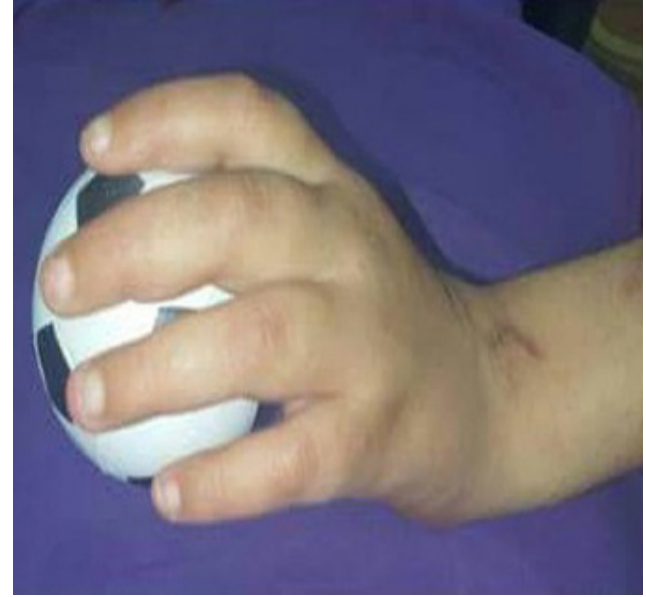

C

Figure 6. (A) The patient is able to maintain intrinsic plus position indicating recovery of the hand intrinsic; (B) Wrist flexion with M3 muscle power; (C) The patient holding a small ball with a good grip.

We do believe that to optimize the functional outcomes of such complex injuries, thumb function is an undeniable priority and its function should be addressed early in the reconstructive plan. Regarding its substantial impact on hand function, any proposed surgical procedure that endangers the thumb function should be omitted, namely, EIP nerve transfer to the motor fascicles of the ulnar nerve. Another paramount deficit to address is the sensation on the ulnar side of the hand.

Direct transfer of the APL nerve to motor fascicles of the ulnar nerve may offer a safe solution for the recovery of intrinsic muscle functions in the combined proximal median and ulnar nerves injury. Simultaneous EIP opponoplasty and using the proximal ulnar nerve as a graft for the median nerve defect optimized the functional outcomes and limited the morbidity. End-to-side transfer of the distal sensory fascicles of the ulnar nerve to the superficial radial nerve is a potential solution for the sensory deficit on the ulnar side of the hand. However, further studies are needed before standardization of this technique.

\section{Article Information}

*Correspondence: Samir Ghoraba, IMRCS, MSC

Department of Plastic and Reconstructive Surgery, Tanta University, Gharbia, Egypt; Email: samir.ghoraba@yahoo.com

Received: Aug. 02, 2017; Accepted: Oct. 12, 2017; Published: Jan. 31, 2018

\section{DOI: 10.24983/scitemed.imj.2018.00051}

Copyright (c) 2018 The Author(s). This is an open-access article distributed under the terms of the Creative Commons Attribution 4.0 International License (CC-BY).

\section{Funding: None}

\section{Conflict of Interest: None}

\section{Acknowledgements}

The aurhors are delighted to acknowledge Moatz Abdelkader for his proofreading and linguistic contributions to this work.

\section{Keywords}

Intrinsic function; median nerve; nerve transfer; opponoplasty; ulnar nerve.

\section{References}

1. Vastamäki M, Kallio PK, Solonen KA. The results of secondary microsurgical repair of ulnar nerve injury. Journal of Hand Surgery 1993;18(3):323-326.

2. Novak CB, Anastakis DJ, Beaton DE, Mackinnon SE, Katz J. Biomedical and psychosocial factors associated with disability after peripheral nerve injury. Journal of Bone and Joint Surgery 2011;93(10):929-936.

3. Haase SC, Chung KC. Anterior interosseous nerve transfer to the motor branch of the ulnar nerve for high ulnar nerve injuries. Annals of Plastic Surgery 2002;49(3):285-290.

4. Brown JM, Yee A, MacKinnon SE. Distal median to ulnar nerve transfers to restore ulnar motor and sensory function within the hand: technical nuances. Neurosurgery 2009;65(5):966-977.

5. Phillips BZ, Franco MJ, Yee A, Tung TH, Mackinnon SE, Fox IK. Direct radial to ulnar nerve transfer to restore intrinsic muscle function in combined proximal median and ulnar nerve injury: case report and surgical technique. Journal of Hand Surgery 2014;39(7):1358-1362. 